\title{
Collocation Technique for Numerical Solution of Integral Equations with Certain Orthogonal Basis Function in Interval $[0,1]$
}

\author{
0. L. Babasola1, I. Irakoze ${ }^{2}$ \\ ${ }^{1}$ University of Ilorin, Ilorin, Nigeria \\ ${ }^{2}$ Université du Burundi, Bujumbura, Burundi \\ Email: babasolaoluwatosin@yahoo.com,irene.irakoze@aims-cameroon.org
}

How to cite this paper: Babasola, O.L. and Irakoze, I. (2017) Collocation Technique for Numerical Solution of Integral Equations with Certain Orthogonal Basis Function in Interval [0, 1]. Open Access Library Journal, 4: e4050. https://doi.org/10.4236/oalib.1104050

Received: October 21, 2017

Accepted: December 12, 2017

Published: December 15, 2017

Copyright ( $\odot 2017$ by authors and Open Access Library Inc.

This work is licensed under the Creative Commons Attribution International License (CC BY 4.0).

http://creativecommons.org/licenses/by/4.0/ (c) (i) Open Access

\begin{abstract}
This paper is concerned with the construction of a class of polynomial orthogonal with respect to the weight function $w(x)=1-x^{2}$ over the interval $[0,1]$. The zeros of these polynomials were employed as points of collocation for the orthogonal collocation technique in the solution of integral equations. The method is illustrated with some numerical examples and the results obtained show that the method is effective.
\end{abstract}

\section{Subject Areas \\ Integral Equation, Numerical Mathematics}

\section{Keywords}

Collocation Method, Integral Equations, Orthogonal Polynomials

\section{Introduction}

Many problems arising in mathematics and in particular, applied mathematics can be formulated into two distinct but connected ways: differential equations and integral equations. Over the years, much emphasis has been placed on the solution of differential equations (ordinary differential equations and partial differential equations) more than the solution of integral equations because one may easily accept that the solution of integral equations are more tasking to obtain compared to the differential equations.

According to [1], Integral equations can be used as the mathematical model in which many physical problems are modelled. The numerical solution of such 
integral equations has been studied by various authors and in recent years, great works have been focused on the development of more advanced and efficient methods for integral equations as they have several applications.

Integral equations can be applied in the radioactive transfer and oscillation problems such as oscillation of string, axle and membrane [1]. Recently, the applications of integral equations have become prominent. However, mathematicians have so far devoted their attention mainly into two peculiarly types of integral equation: the linear equations of the first and second kinds.

An Integral equation is an equation in which the unknown function appears under one or more integral sign [2]. The standard integral equations of the form

$$
\int_{\alpha}^{\beta} K(x, t) g(t) \mathrm{d} t=\varphi(x)
$$

and

$$
\lambda \int_{\alpha}^{\beta} K(x, t) g(t) \mathrm{d} t=g(x)+\varphi(x)
$$

are known as the linear Fredholm integral equations of the first and second kinds respectively. In each case, $g(x)$ is the unknown function and it occurs to the first degree while the kernel $K(x, t)$ and $\varphi(x)$ are the known functions. If the constant $\beta$ in (1.1) and (1.2) is replaced by $x$ (the variable of integration), then the equations become Volterra integral equations. Thus, the integral equations of the form

$$
\int_{a}^{x} K(x, t) g(t) \mathrm{d} t=\varphi(x)
$$

and

$$
\lambda \int_{a}^{x} K(x, t) g(t) \mathrm{d} t=g(x)+\varphi(x)
$$

are called the Volterra integral equations of the first and second kinds.

If $\varphi(x)=0$ in (1.3) and (1.4), then we say the equation is homogeneous, otherwise nonhomogeneous.

\section{Literature Review}

Collocation method involves evaluating of approximate solution in a suitable set of functions called basis function or trial solution. This method for obtaining the approximate solution to an integral equation has its origin in the 1930s when [3] consider an integral equation using the line collocation procedure. [4] used orthogonal collocation to solve a boundary value problems where he developed the set of orthogonal polynomials using both the boundary conditions and the roots of the polynomials as the collocation points.

Recently, many researchers have developed the numerical method to obtain the solution to an integral equation using several well known polynomials and in particular, orthogonal polynomials.

[5] obtained the numerical solution of the Volterra integral equation of second kind using the Gelerkin method and he used the Hermite polynomials as 
the basis function. Similarly, [6] explore the solution of both the linear and nonlinear Volterra integral equation using the Gelerkin method but they used the Hermite and Chebyshev polynomials as the basis function. [7] also considered the first kind boundary integral equation and obtained its numerical solution by the means of attenuation factors. [8] did a work by using an extrapolation techniques and collocation method for some integral equations. [9] obtained a numerical solution of the integral equation of second kind and he compared the error with that of analytics solution. [10] uses the numerical expansion methods to solve Fredholm-Volterra linear integral equation by interpolation and quadrature rules while [11] formulated and use the collocation technique to obtain the numerical solution of the fredholm second kind integral equation.

However in this paper, the orthogonal collocation techniques will be use to obtain the numerical solution of linear integral equation and the zeros of a constructed orthogonal polynomials will be used as the points of collocation. Thereafter, the result obtained will be compare with the analytic solution to show that the method is effective and accurate.

\section{Construction of Orthogonal Polynomials}

Let $\varphi_{n}(x)$ be a polynomial of exact degree $n$, then $\varphi_{n}$ is said to be orthogonal with respect to a weight function $w(x)$ within the interval $[\alpha, \beta] \in \mathbb{R}$ with $\alpha<\beta$ if

$$
\int_{\alpha}^{\beta} \phi_{m}(x) \phi_{n}(x) w(x) \mathrm{d} x=\delta_{m n},
$$

with $\delta_{m n}$ is the Kronecker symbol defined by:

$$
\delta_{m n}= \begin{cases}1 & \text { if } m=n, \\ 0 & \text { if } m \neq n .\end{cases}
$$

The weight function $w(x)$ should be continuous and also positive on $[\alpha, \beta]$ such that the moments

$$
\int_{\alpha}^{\beta} w(x) x^{n} \mathrm{~d} x, \quad n \in \mathbb{N}
$$

exists and finite. Then

$$
\left\langle\phi_{m}, \phi_{n}\right\rangle=\int_{\alpha}^{\beta} \phi_{m}(x) \phi_{n}(x) w(x) \mathrm{d} x
$$

defines the inner product of the polynomial $\phi_{m}$ and $\phi_{n}$.

We shall adopt the weight function $w(x)=1-x^{2}$ in the interval $[0,1]$. Hence, we use the property below to construct our basis function.

$$
\begin{gathered}
\phi_{n}(x)=\sum_{r=0}^{n} C_{r}^{(n)} x^{r} \\
\left\langle\phi_{m}, \phi_{n}\right\rangle=0 \\
\phi_{n}(1)=1
\end{gathered}
$$

For $\phi_{0}(x)$, we have 


$$
\begin{gathered}
\phi_{0}(x)=\sum_{r=0}^{0} C_{r}^{(0)} x^{r}=C_{0}^{(0)} \\
\phi_{0}(1)=C_{0}^{(0)}=1 \\
\phi_{0}(x)=1
\end{gathered}
$$

For $\phi_{1}(x)$, we have

$$
\phi_{1}(x)=\sum_{r=0}^{1} C_{r}^{(1)} x^{r}=C_{0}^{(1)}+C_{1}^{(1)} x .
$$

When $x=1$,

$$
\begin{gathered}
\phi_{1}(1)=C_{0}^{(1)}+C_{1}^{(1)}=1 \\
\left\langle\phi_{0}, \phi_{1}\right\rangle=\int_{0}^{1}\left(1-x^{2}\right) \phi_{0}(x) \phi_{1}(x) \mathrm{d} x=0 \\
=\int_{0}^{1}\left(1-x^{2}\right)\left(C_{0}^{(1)}+C_{1}^{(1)} x\right) \mathrm{d} x=0 \\
=\frac{2}{3} C_{0}^{(1)}+\frac{1}{4} C_{1}^{(1)}=0
\end{gathered}
$$

solving (3.5) and (3.6), we obtain

$$
C_{0}^{(1)}=-\frac{3}{5}, \quad C_{1}^{(1)}=\frac{8}{5}
$$

Hence,

$$
\phi_{1}(x)=\frac{1}{5}(8 x-3)
$$

Similarly,

For $\phi_{2}(x)$, we have

$$
\phi_{2}(x)=\sum_{r=0}^{2} C_{r}^{(2)} x^{r}=C_{0}^{(2)}+C_{1}^{(2)} x+C_{2}^{(2)} x^{2} .
$$

For $x=1$, we obtain

$$
\phi_{2}(1)=C_{0}^{(2)}+C_{1}^{(2)}+C_{2}^{(2)}=1
$$

and

$$
\begin{gathered}
\left\langle\phi_{0}, \phi_{2}\right\rangle=\int_{0}^{1}\left(1-x^{2}\right) \phi_{0}(x) \phi_{2}(x) \mathrm{d} x=0 \\
\int_{0}^{1}\left(1-x^{2}\right)\left(C_{0}^{(2)}+C_{1}^{(2)} x+C_{2}^{(2)} x^{2}\right) \mathrm{d} x=0 \\
\frac{2}{3} C_{0}^{(2)}+\frac{1}{4} C_{1}^{(2)}+\frac{2}{15} C_{2}^{(2)}=0 \\
\left\langle\phi_{1}, \phi_{2}\right\rangle=\int_{0}^{1}\left(1-x^{2}\right) \phi_{1}(x) \phi_{2}(x) \mathrm{d} x=0 \\
\frac{1}{5} \int_{0}^{1}\left(1-x^{2}\right)(8 x-3)\left(C_{0}^{(2)}+C_{1}^{(2)} x+C_{2}^{(2)} x^{2}\right) \mathrm{d} x=0 \\
\frac{19}{300} C_{1}^{(2)}+\frac{4}{75} C_{2}^{(2)}=0
\end{gathered}
$$

solving (3.8), (3.9) and (3.10), we obtain 


$$
C_{0}^{(2)}=\frac{11}{26}, \quad C_{1}^{(2)}=-\frac{80}{26}, \quad C_{2}^{(2)}=\frac{95}{26} .
$$

Hence,

$$
\phi_{2}(x)=\frac{1}{26}\left(95 x^{2}-80 x+11\right) .
$$

Following the same procedure,

$$
\begin{gathered}
\phi_{3}(x)=\frac{1}{46}\left(448 x^{3}-595 x^{2}+208 x-15\right) \\
\phi_{4}(x)=\frac{1}{743}\left(21042 x^{4}-38304 x^{3}+22232 x^{2}-4424 x+197\right) \\
\phi_{5}(x)=\frac{1}{4043}\left(352176 x^{5}-815430 x^{4}+6669 x^{3}-229320 x^{2}+29840 x-903\right) \\
\phi_{6}(x)=\frac{1}{22180}\left(6180603 x^{6}-17379648 x^{5}+18440235 x^{4}-9144960 x^{3}\right. \\
\left.+2116935 x^{2}-195264 x+4279\right)
\end{gathered}
$$

\section{Numerical Examples}

We consider here three problems for illustration of the proceeding discourse. For this purpose, we seek approximant of degree 3, 4 and 5 (Tables 1-5).

Example 1 Consider the integral equation

$$
\int_{0}^{1} x e^{x s} y(s) \mathrm{d} s=e^{x}-y(x), \quad \text { whose analytic solution is } y(x)=1 .
$$

Solving with $N=3$ as the degree of approximation, we have

$$
\begin{gathered}
\int_{0}^{1} x e^{x s} \sum_{r=0}^{3} a_{r} \phi_{r}(s) \mathrm{d} s=e^{x}-\sum_{r=0}^{3} a_{r} \phi_{r}(x) \\
\int_{0}^{1} x e^{x s}\left\{a_{0}+a_{1}\left(\frac{8}{5} s-\frac{3}{5}\right)+a_{2}\left(\frac{95}{26} s^{2}-\frac{80}{26} s+\frac{11}{26}\right)\right. \\
\left.+a_{3}\left(\frac{224}{23} s^{3}-\frac{595}{46} s^{2}+\frac{104}{23} s-\frac{15}{46}\right)\right\} \mathrm{d} s \\
=e^{x}-\left\{a_{0}+a_{1}\left(\frac{8}{5} x-\frac{3}{5}\right)+a_{2}\left(\frac{95}{26} x^{2}-\frac{80}{26} x+\frac{11}{26}\right)\right. \\
\left.+a_{3}\left(\frac{224}{23} x^{3}-\frac{595}{46} x^{2}+\frac{104}{23} x-\frac{15}{46}\right)\right\}
\end{gathered}
$$

This gives

$$
\begin{aligned}
& \frac{1}{2990 x^{3}}\left\{29120 x^{6} a_{3}+10925 x^{5} a_{2}-38675 x^{5} a_{3}+2990 e^{x} x_{3}\left(a_{0}+a_{1}+a_{2}+a_{3}\right)\right. \\
& +4784 x^{4} a_{1}-9200 x^{4} a_{2}+13520 x^{4} a_{3}-4784 e^{x} x^{2} a_{1}-12650 e^{x} x^{2} a_{2} \\
& -23530 e^{x} x^{2} a_{3}+21850 e^{x} x a_{2}+97370 e^{x} x a_{3}+4784 x^{2} a_{1}-9200 x^{2} a_{2} \\
& \left.+13520 x^{2} a_{3}-174720 e^{x} a_{3}-21850 x a_{2}+77350 x a_{3}+174720 a_{3}\right\}=e^{x}
\end{aligned}
$$

As there are four unknown coefficients in this equation, we shall collocate at 
the zeros of the fourth degree polynomial earlier constructed, This results into the linear system of equations:

$$
\begin{aligned}
& 1.063634023 a_{0}-0.4880435909 a_{1}+0.2538803066 a_{2}-0.0901090390 a_{3} \\
& =1.063634023 \\
& 1.338950765 a_{0}-0.0520191234 a_{1}-0.1239293627 a_{2}+0.1566142614 a_{3} \\
& =1.338950765 \\
& 1.821140964 a_{0}+0.5886120730 a_{1}+0.0023209561 a_{2}-0.1022283745 a_{3} \\
& =1.821140964 \\
& 2.380518708 a_{0}+1.221493409 a_{1}+0.7117809561 a_{2}+0.3397428175 a_{3} \\
& =2.380518708
\end{aligned}
$$

Solving the equations above, we have

$$
a_{0}=0.9999999999, a_{1}=a_{2}=a_{3}=0
$$

Thus, the approximate solution,

$$
y_{3}(x)=0.9999999999
$$

Next, we seek an approximant of degree $N=4$ and for this, we shall engage the zeros of the fifth degree orthogonal polynomial constructed. This leads to the equations

$$
\begin{aligned}
& 0.9-0.4574733239 a_{0}+0.7737959868 a_{1}-0.1951129745 a_{2} \\
& +0.2172348194 a_{3}-0.02283297131 a_{4}=0.7695433192 \\
& 0.9-0.2916717999 a_{0}+0.5416738531 a_{1}+0.3668860626 a_{2} \\
& -0.07218775521 a_{3}+0.1350402311 a_{4}=0.5208410331 \\
& 0.9-0.0477684829 a_{0}+0.2002092093 a_{1}+0.366886062 a_{2} \\
& +0.11103526617 a_{3}-0.053569893 a_{4}=0.1549860577 \\
& 0.90 .2065322412 a_{0}-0.1558118044 a_{1}+0.098736803 a_{2} \\
& +0.252593850 a_{3}+0.194777949 a_{4}=-0.226465028 \\
& 0.90 .4057861632 a_{0}-0.4347672951 a_{1}-0.441575320 a_{2} \\
& -0.284119350 a_{3}-0.10098142 a_{4}=-0.5253459117
\end{aligned}
$$

Solving the equations,

$$
a_{0}=1.000000, a_{1}=a_{2}=a_{3}=a_{4}=0
$$

Therefore

$$
y_{4}(x)=1
$$

Similarly, for a fifth degree approximant, we use the zeros of the sixth degree orthogonal polynomial as our point of collocation to also get $y_{5}(x)=1$ as the desired approximation.

Example 2 Consider

$$
\int_{0}^{1}(x+s) y(s) \mathrm{d} s=y(x)-\frac{3}{2} x+\frac{5}{6}, \quad \text { whose analytic solution is } y(x)=-1+x .
$$

For a third degree approximant of $y(x)$, we have 


$$
\begin{aligned}
& \int_{0}^{1}(x+s)\left\{\left(a_{0}-\frac{3}{5} a_{1}+\frac{11}{26} a_{2}-\frac{15}{46} a_{3}\right)+\left(\frac{8}{5} a_{1}-\frac{80}{26} a_{2}+\frac{208}{46} a_{3}\right) s\right. \\
& \left.+\left(\frac{95}{26} a_{2}-\frac{595}{46} a_{3}\right) s^{2}+\frac{224}{23} a_{3} s^{3}\right\} \mathrm{d} s \\
& =\left\{\left(a_{0}-\frac{3}{5} a_{1}+\frac{11}{26} a_{2}-\frac{15}{46} a_{3}\right)+\left(\frac{8}{5} a_{1}-\frac{80}{26} a_{2}+\frac{208}{46} a_{3}\right) x\right. \\
& \left.+\left(\frac{95}{26} a_{2}-\frac{595}{46} a_{3}\right) x^{2}+\frac{224}{23} a_{3} x^{3}-\frac{3}{2} x+\frac{5}{6}\right\}
\end{aligned}
$$

This gives

$$
\begin{aligned}
& \frac{1061}{2760} a_{3}-\frac{308}{69} x a_{3}-\frac{101}{312} a_{2}-\frac{124}{39} x a_{2}+\frac{5}{6} a_{1}-\frac{7}{5} x a_{1}-\frac{1}{2} a_{0}+x a_{0} \\
& -\frac{95}{26} x^{2} a_{2}+\frac{595}{46} x^{2} a_{3}-\frac{224}{23} a_{3} x^{3}+\frac{3}{2} x-\frac{5}{6}=0
\end{aligned}
$$

collocating this we obtain the system of equations

$$
\begin{aligned}
- & 0.4383086312 a_{0}+0.7469654169 a_{1}-0.1414769314 a_{2}+0.1559852267 a_{3} \\
= & 0.7407962800 \\
- & 0.2081137040 a_{0}+0.4246925189 a_{1}+0.2930318285 a_{2}-0.0586727298 a_{3} \\
= & 0.3955038893 \\
& 0.0994632079 a_{0}-0.0059151577 a_{1}+0.2692355947 a_{2}+0.258737951 a_{3} \\
= & -0.0658614785 \\
& 0.3673184086 a_{0}-0.3809124389 a_{1}-0.314663883 a_{2}-0.111138066 a_{3} \\
= & -0.4676442797
\end{aligned}
$$

We solve these to obtain

$$
\begin{aligned}
& a_{0}=-0.6249999992, a_{1}=0.6250000003, \\
& a_{2}=1.211249628 \times 10^{-10}, a_{3}=-2.268186446 \times 10^{-10}
\end{aligned}
$$

and hence

$$
\begin{aligned}
y_{3}(x)= & -0.9999999992+0.9999999986 x+3.376421840 \times 10^{-9} x^{2} \\
& -2.209016365 \times 10^{-9} x^{3} .
\end{aligned}
$$

For a quartic approximation of $y(x)$ we obtain by using the zeros of the fifth degree polynomial and we have

$$
\begin{aligned}
y_{4}(x)= & -1.000000001+1.000000002 x-2.101878150 \times 10^{-8} x^{2} \\
& +3.718048589 \times 10^{-8} x^{3}-1.995546318 \times 10^{-8} x^{4}
\end{aligned}
$$

Similarly, for an approximation of degree 5 we obtained

$$
\begin{aligned}
y_{5}(x)= & -0.9999999981+0.9999999995 x+2.545793296 \times 10^{-8} x^{2} \\
& -7.244844114 \times 10^{-8} x^{3}+9.516056846 \times 10^{-8} x^{4} \\
& -4.631806729 \times 10^{-8} x^{5}
\end{aligned}
$$

Example 3 Consider the integral equation

$$
y(x)=1+\int_{0}^{1} x^{2} s^{3} y(s) \mathrm{d} s, \quad \text { whose analytic solution is } y(x)=1+\frac{3}{10} x^{2} .
$$


By seeking a cubic approximation to $y(x)$ we have

$$
\begin{gathered}
1+\int_{0}^{1} x^{2} s^{3} \sum_{r=0}^{3} a_{r} \phi_{r}(s) \mathrm{d} s=\sum_{r=0}^{3} a_{r} \phi_{r}(x) \\
1+\int_{0}^{1} x^{2} s^{3}\left\{\left(a_{0}-\frac{3}{5} a_{1}+\frac{11}{26} a_{2}-\frac{15}{46} a_{3}\right)+\left(\frac{8}{5} a_{1}-\frac{80}{26} a_{2}+\frac{208}{46} a_{3}\right) s\right. \\
\left.+\left(\frac{95}{26} a_{2}-\frac{595}{46} a_{3}\right) s^{2}+\frac{224}{23} a_{3} s^{3}\right\} \mathrm{d} s \\
=\left(a_{0}-\frac{3}{5} a_{1}+\frac{11}{26} a_{2}-\frac{15}{46} a_{3}\right)+\left(\frac{8}{5} a_{1}-\frac{80}{26} a_{2}+\frac{208}{46} a_{3}\right) x \\
+\left(\frac{95}{26} a_{2}-\frac{595}{46} a_{3}\right) x^{2}+\frac{224}{23} a_{3} x^{3}
\end{gathered}
$$

That is

$$
\begin{aligned}
& \frac{35861}{2760} x^{2} a_{3}-\frac{1109}{312} x^{2} a_{2}+\frac{17}{100} x^{2} a_{1}+\frac{1}{4} x^{2} a_{0}-a_{0}+\frac{3}{5} a_{1}-\frac{11}{26} a_{2} \\
& +\frac{15}{4} a_{3}-\frac{8}{5} x a_{1}+\frac{40}{13} x a_{2}-\frac{104}{23} x a_{3}-\frac{224}{23} a_{3} x^{3}=-1
\end{aligned}
$$

collocating this at the four points, we have the linear system

$$
\begin{aligned}
& -0.9990485438 a_{0}+0.5019408002 a_{1}-0.2467850828 a_{2}+0.0942975884 a_{3}=-1 \\
& -0.9787005976 a_{0}+0.1474655200 a_{1}+0.1722009453 a_{2}-0.1289571472 a_{3}=-1 \\
& -0.9101609656 a_{0}-0.2980505892 a_{1}+0.1440984716 a_{2}+0.186615570 a_{3}=-1 \\
& -0.8119396945 a_{0}-0.6598284463 a_{1}-0.4282366757 a_{2}-0.175869988 a_{3}=-1
\end{aligned}
$$

We solve this to have

$$
\begin{aligned}
& a_{0}=1.060000000, a_{1}=0.1578947373, \\
& a_{2}=0.08210526282, a_{3}=1.260103578 \times 10^{-10}
\end{aligned}
$$

so that

$$
y_{3}(x)=0.9999999996+2.4 \times 10^{-9} x+0.2999999972 x^{2}+1.227231311 \times 10^{-9} x^{3}
$$

is our desired approximant of $y(x)$

Similarly, for the quartic and quintic approximant of $y(x)$, we obtained respectively

$$
\begin{aligned}
y_{4}(x)= & 1.0000+9.0 \times 10^{-10} x+0.3000000052 x^{2}-9.297606497 \times 10^{-9} x^{3} \\
& +5.11785260 \times 10^{-8} x^{4} \\
y_{5}(x)= & 1.000000000-6.8 \times 10^{-9} x+0.3000000373 x^{2}-8.393008256 \times 10^{-8} x^{3} \\
+ & 8.191152506 \times 10^{-8} x^{4}-2.880832986 \times 10^{-8} x^{5}
\end{aligned}
$$

\section{Conclusion}

A method for the numerical solution of integral equations has been presented. The method employs the idea of collocation and it uses a class of orthogonal polynomials with respect to the weight function $w(x)=1-x^{2}$ over the interval [0, 1]. The zeros or roots of the orthogonal polynomials were chosen as collocation 
Table 1. Numerical results for 1 .

\begin{tabular}{ccccc}
\hline$x$ & Exact & Approximate & Approximate & Approximate \\
\hline & Solution & Solution & Solution & Solution \\
\hline 0.01 & & $N=3$ & $N=4$ & $N=5$ \\
0.02 & 1.000000 & 0.999999999 & 1.000000 & 1.000000 \\
0.03 & 1.000000 & 0.999999999 & 1.000000 & 1.000000 \\
0.04 & 1.000000 & 0.999999999 & 1.000000 & 1.000000 \\
0.05 & 1.000000 & 0.999999999 & 1.000000 & 1.000000 \\
0.06 & 1.000000 & 0.999999999 & 1.000000 & 1.000000 \\
0.07 & 1.000000 & 0.999999999 & 1.000000 & 1.000000 \\
0.08 & 1.000000 & 0.999999999 & 1.000000 & 1.000000 \\
0.09 & 1.000000 & 0.999999999 & 1.000000 & 1.000000 \\
0.10 & 1.000000 & 0.999999999 & 1.000000 & 1.000000 \\
\hline
\end{tabular}

Table 2. Error results for 1.

\begin{tabular}{cccc}
\hline$X$ & Error, $N=3$ & Error, $N=4$ & Error, $N=5$ \\
\hline 0.01 & $1.00 \mathrm{e}-09$ & $0.00 \mathrm{e}+00$ & $0.00 \mathrm{e}+00$ \\
0.02 & $1.00 \mathrm{e}-09$ & $0.00 \mathrm{e}+00$ & $0.00 \mathrm{e}+00$ \\
0.03 & $1.00 \mathrm{e}-09$ & $0.00 \mathrm{e}+00$ & $0.00 \mathrm{e}+00$ \\
0.04 & $1.00 \mathrm{e}-09$ & $0.00 \mathrm{e}+00$ & $0.00 \mathrm{e}+00$ \\
0.05 & $1.00 \mathrm{e}-09$ & $0.00 \mathrm{e}+00$ & $0.00 \mathrm{e}+00$ \\
0.06 & $1.00 \mathrm{e}-09$ & $0.00 \mathrm{e}+00$ & $0.00 \mathrm{e}+00$ \\
0.07 & $1.00 \mathrm{e}-09$ & $0.00 \mathrm{e}+00$ & $0.00 \mathrm{e}+00$ \\
0.08 & $1.00 \mathrm{e}-09$ & $0.00 \mathrm{e}+00$ & $0.00 \mathrm{e}+00$ \\
0.09 & $1.00 \mathrm{e}-09$ & $0.00 \mathrm{e}+00$ & $0.00 \mathrm{e}+00$ \\
0.10 & $1.00 \mathrm{e}-09$ & $0.00 \mathrm{e}+00$ & $0.00 \mathrm{e}+00$ \\
\hline
\end{tabular}

Table 3. Numerical results for 2 .

\begin{tabular}{ccccc}
\hline$X$ & Exact & Approximate & Approximate & Approximate \\
\hline & Solution & Solution & Solution & Solution \\
\hline 0.01 & -0.99000000 & -0.9899999992 & -0.9900000010 & -0.99000000 \\
0.02 & -0.98000000 & -0.9799999992 & -0.9800000010 & -0.98000000 \\
0.03 & -0.97000000 & -0.9699999992 & -0.9700000010 & -0.97000000 \\
0.04 & -0.96000000 & -0.9599999993 & -0.9600000010 & -0.96000000 \\
0.05 & -0.95000000 & -0.9499999993 & -0.9500000010 & -0.95000000 \\
0.06 & -0.94000000 & -0.9399999993 & -0.9400000010 & -0.94000000 \\
0.07 & -0.93000000 & -0.9299999993 & -0.9300000010 & -0.93000000 \\
0.08 & -0.92000000 & -0.9199999993 & -0.9200000010 & -0.92000000 \\
0.09 & -0.91000000 & -0.9099999993 & -0.9100000010 & -0.91000000 \\
0.10 & -0.90000000 & -0.8999999993 & -0.9000000010 & -0.90000000 \\
\hline
\end{tabular}


Table 4. Error results for 2. $\quad 7.00 \mathrm{e}-10 \quad 1.00 \mathrm{e}-10 \quad 0.00 \mathrm{e}+00$

\begin{tabular}{cccc}
\hline$x$ & Error, $N=3$ & Error, $N=4$ & Error, $N=5$ \\
\hline 0.01 & $8.00 \mathrm{e}-10$ & $1.00 \mathrm{e}-10$ & $0.00 \mathrm{e}+00$ \\
0.02 & $8.00 \mathrm{e}-10$ & $1.00 \mathrm{e}-10$ & $0.00 \mathrm{e}+00$ \\
0.03 & $8.00 \mathrm{e}-10$ & $1.00 \mathrm{e}-10$ & $0.00 \mathrm{e}+00$ \\
0.04 & $7.00 \mathrm{e}-10$ & $1.00 \mathrm{e}-10$ & $0.00 \mathrm{e}+00$ \\
0.05 & $7.00 \mathrm{e}-10$ & $1.00 \mathrm{e}-10$ & $0.00 \mathrm{e}+00$ \\
0.06 & $7.00 \mathrm{e}-10$ & $1.00 \mathrm{e}-10$ & $0.00 \mathrm{e}+00$ \\
0.07 & $7.00 \mathrm{e}-10$ & $1.00 \mathrm{e}-10$ & $0.00 \mathrm{e}+00$ \\
0.08 & $7.00 \mathrm{e}-10$ & $1.00 \mathrm{e}-10$ & $0.00 \mathrm{e}+00$ \\
0.09 & $7.00 \mathrm{e}-10$ & $1.00 \mathrm{e}-10$ & $0.00 \mathrm{e}+00$ \\
0.10 & $7.00 \mathrm{e}-10$ & $1.00 \mathrm{e}-10$ & $0.00 \mathrm{e}+00$ \\
\hline
\end{tabular}

Table 5. Numerical results for 3 .

\begin{tabular}{ccccc}
\hline$x$ & Exact & Approximate & Approximate & Approximate \\
\hline & Solution & Solution, & Solution, & Solution, \\
\hline 0.01 & 1.0000300 & 1.0000300 & 1.0000300 & $N=5$ \\
0.02 & 1.0001200 & 1.0001200 & 1.0001200 & 1.0000300 \\
0.03 & 1.0002700 & 1.0002700 & 1.0002700 & 1.0002700 \\
0.04 & 1.0004800 & 1.0004800 & 1.0004800 & 1.0004800 \\
0.05 & 1.0007500 & 1.0007500 & 1.0007500 & 1.0007500 \\
0.06 & 1.0010800 & 1.0010800 & 1.0010800 & 1.0010800 \\
0.07 & 1.0014700 & 1.0014700 & 1.0014700 & 1.0014700 \\
0.08 & 1.0019200 & 1.0019200 & 1.0019200 & 1.0019200 \\
0.09 & 1.0024300 & 1.0024300 & 1.0024300 & 1.0024300 \\
0.10 & 1.0030000 & 1.0030000 & 1.0030000 & 1.0030000 \\
\hline
\end{tabular}

points for an orthogonal collocation technique. Three numerical examples were considered to illustrate the proposed method. However, the numerical evidences show that method is effective and gives better approximation solution compared to the one in the literatures.

\section{Acknowledgements}

I wish to acknowledge the contribution of my mentor towards the completion of this paper.

\section{References}

[1] Brunner, H. (2004) Collocation Methods for Volterra Integral and Related Functional Differential Equations. Cambridge University Press, Cambridge. 
https://doi.org/10.1017/CBO9780511543234

[2] Sastry, S.S. (2012) Introductory Methods of Numerical Analysis. PHI Learning Pvt. Ltd., Delhi.

[3] Kantorovich, L.V. (1934) On Approximate Calculation of Some Type of Definite Integrals and Other Applications of the Method of Singularities Extraction. Matematicheskii Sbornik, 41, 235-245.

[4] Villadsen, J. and Stewart, W. (1967) Solution of Boundary-Value Problems by Orthogonal Collocation. Chemical Engineering Science, 22, 1483-1501.

https://doi.org/10.1016/0009-2509(67)80074-5

[5] Rahman, M. M. (2013) Numerical Solutions of Volterra Integral Equations Using Galerkin Method with Hermite Polynomials. Proceedings of the International Conference on Applied Mathematics and Computational Methods in Engineering.

[6] Islam, M.S. and Rahman, M.A. (2013) Solutions of Linear and Nonlinear Volterra Integral Equations Using Hermite and Chebyshev Polynomials. International Journal of Computers \& Technology, 11, 2910-2920.

[7] Reifenberg, M. and Berrut, J.P. (2000) Numerical Solution of Boundary Integral Equations by Means of Attenuation Factors. IMA Journal of Numerical Analysis, 20, 25-46. https://doi.org/10.1093/imanum/20.1.25

[8] Celorrio, R and Sayas, F.J. (2001) Extrapolation Techniques and the Collocation Method for a Class of Boundary Integral Equations. The ANZIAM Journal, 42, 413-437. https://doi.org/10.1017/S1446181100012037

[9] Atkinson, K.E. (1997) The Numerical Solution of Integral Equations of the Second Kind. Cambridge University Press, Cambridge, Vol. 4.

https://doi.org/10.1017/CBO9780511626340

[10] Yusufoglu, E. and Erbas, B. (2008) Numerical Expansion Methods for Solving Fredholm-Volterra Type Linear Integral Equations by Interpolation and Quadrature rules. Kybernetes, 37, 768-785. https://doi.org/10.1108/03684920810876972

[11] Ramm, A. (2009) A Collocation Method for Solving Integral Equations. International Journal of Computing Science and Mathematics, 2, 222-228.

https://doi.org/10.1504/IJCSM.2009.027874 